\title{
Frecuencia del bucle del nervio mentoniano en tomografía computarizada de haz cónico en el Hospital Nacional Cayetano Heredia
}

Frecuency of the mentonian nerve loop in computed cone beam computed tomography at the Hospital Nacional Cayetano Heredia

\author{
Roger Condori Cruz ${ }^{1, a}$, Alfredo Yupanqui Pellanne ${ }^{2,3, b, c}$, Alexis Evangelista-Alva ${ }^{2, c, f}$, Milushka Miroslava \\ Quezada Márquez 2,a,c,d,e
}

\section{RESUMEN}

Objetivo: El propósito de este estudio fue determinar la frecuencia del bucle del nervio mentoniano en pacientes del Servicio de Radiología Oral y Maxilofacial de la Unidad de Apoyo al Diagnóstico Estomatológico del Departamento de Odontoestomatología del Hospital Cayetano Heredia, periodo 2016-2017. Material y Métodos: El presente estudio fue de tipo descriptivo, retrospectivo y de corte transversal. Se analizaron 181 tomografías computarizadas de haz cónico seleccionadas de acuerdo a los criterios de inclusión y exclusión. Se evaluó el bucle del nervio mentoniano respecto a su presencia o ausencia y su respectiva caracterización. Para el análisis, se usó la estadística descriptiva y la prueba de Chi Cuadrado a un intervalo de confianza al 95\%, y un nivel de significancia de 0,05. Resultados: Se tuvieron 97 hallazgos del bucle del nervio mentoniano que representan el 53,6\% pacientes, de los cuales el 74,2\% fueron del sexo femenino. Predominaron los hallazgos en el grupo de 20 a 29 años con un $28,9 \%$. De acuerdo a la zona de distribución la mayoría estuvieron en el lado izquierdo $(23,8 \%)$. En cuanto a la longitud, se encontró una mínima de $0,12 \mathrm{~mm}$ y una máxima de 7,62 mm. Conclusiones: Predominaron los hallazgos en el sexo femenino y en el lado izquierdo, asimismo se verificó como medida media para el bucle del nervio mentoniano de $3,04 \mathrm{~mm}$; estos resultados servirán posteriormente para implementar la búsqueda radiográfica de rutina del mencionado paquete vásculonervioso, previo a los tratamientos quirúrgicos en la zona del foramen mental.

PALABRAS CLAVE: Agujero mentoniano, variación anatómica, tomografía computarizada de haz cónico.

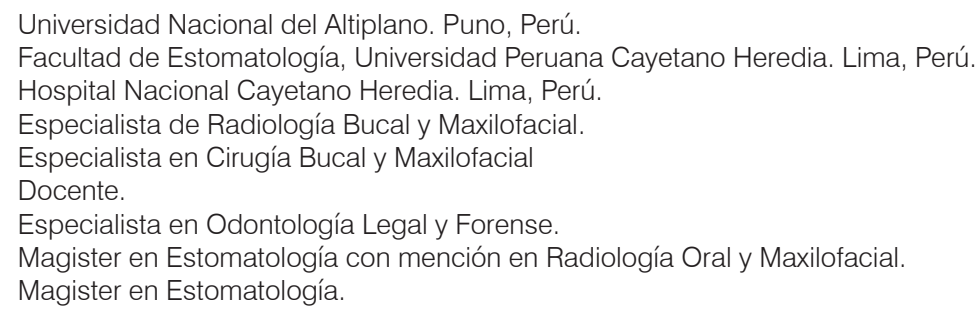




\section{SUMMARY}

Objective: This study purpose is to determine mental nerve loop frequency in patients of the Oral and Maxillofacial Radiology Service of Stomatological Diagnostic Support Unit of Odontoestomatology Department of Cayetano Heredia Hospital, 2016-2017 period. Material and Methods: A descriptive, retrospective and cross-sectional type was present study. We analyzed 181 cone beam computed tomographies selected according to inclusion and exclusion criteria, and evaluated mental nerve loop with respect to its presence or absence and its respective characterization. For the analysis, descriptive statistics and Chi square test were used at a $95 \%$ confidence interval, and a significance level of 0.05. Results: There were 97 findings of mentonian nerve loop that represent 53.6\% patients, of which $74.2 \%$ were female. The findings predominated in the group of 20 to 29 years with $28.9 \%$. According to the distribution area, the majority were on the left side $(23.8 \%)$. As for the length, a minimum of $0.12 \mathrm{~mm}$ and a maximum of $7.62 \mathrm{~mm}$ was found. Conclusions: The findings prevailed in the female sex and in the left side, likewise it was verified as a mean measurement for the $3.04 \mathrm{~mm}$ mental nerve loop, these results will later serve to implement routine radiographic search of the mentioned neurovascular package, previous to surgical treatments in mental foramen area.

\section{KEYWORDS: Mental hole, anatomic variation, cone beam computed tomography.}

\section{INTRODUCCIÓN}

El nervio mentoniano (NM) es un nervio somático aferente que proporciona sensación al labio, la barbilla y el tejido blando gingival. Es la rama terminal del nervio mandibular, que es la tercera división del $\mathrm{V}$ par craneal. El NM sale de la mandíbula a través del foramen mental (FM), luego se divide en 3 ramas profundas en el músculo triangular de los labios y suministra inervación a la piel del área del mentón, membranas mucosas, encía y la piel del labio inferior, también da inervación a los tejidos de la zona del canino e incisivos (1). La literatura anatómica ha descrito la región interforaminal de la mandíbula como poseedor de un NM con un camino que crea un bucle del nervio mentoniano (BNM) antes de entrar en el agujero mentoniano y otra división en este punto (anterior al BNM) como el nervio incisivo (2).

El conducto mandibular que contiene el haz neurovascular alveolar inferior atraviesa el FM anteriormente (figura 1), su parte final del nervio alveolar inferior (NAI) o dentario inferior pasa a veces por debajo del borde inferior y la pared anterior del agujero mentoniano $\mathrm{y}$, después de dar una rama incisiva, en la región donde se divide en los conductos mentales e incisivos, se curva hacia atrás para entrar en el agujero y emerge a los tejidos blandos convirtiéndose en el NM. Esta característica anatómica también es conocidas como "bucle anterior" del nervio alveolar inferior $(3,4)$.

El tratamiento quirúrgico se puede complicar por la extensión desconocida del BNM, las alteraciones sensoriales de la región mental pueden surgir después de procedimientos quirúrgicos en la región del FM. Un problema relativamente común es el daño del NAI y el NM. La incidencia de alteraciones sensoriales permanentes en el labio inferior después de intervenciones quirúrgicas en el área del FM se calcula entre el $7 \%$ y el $10 \%(5,6)$. Las complicaciones tales como pérdida de la sensación del labio y del mentón pueden dar lugar a la mordedura del labio, alteración del habla y disminución de la retención salival (7).

Así, es fundamental conocer la anatomía quirúrgica de esta región, para la rehabilitación con implantes dentales, injertos óseos o cirugías de los ápices dentarios, evitando así lesionar estructuras que la comprometen (8). En ese sentido, el examen radiográfico preoperatorio es esencial para realizar los procedimientos quirúrgicos, pues el profesional debe estar familiarizado con las dimensiones, forma de los maxilares y la ubicación de las estructuras anatómicas.

La radiografía panorámica (RP) proporciona información bidimensional básica, mientras que la 
tomografía computarizada de haz cónico (TCHC) nos brinda una determinación tridimensional, sin magnificación ni sobreposición de estructuras, además la RP tienen limitaciones severas para la evaluación de estructuras anatómicas y hueso disponible $(9,10)$ llevando a falsos positivos de diagnóstico del BNM (7).

El objetivo de esta investigación fue determinar la frecuencia y caracterización del bucle del nervio mentoniano en tomografía computarizada de haz cónico en el Hospital Nacional Cayetano Heredia durante el periodo 2016 al 2017.

\section{MATERIAL Y MÉTODOS}

El trabajo fue de tipo descriptivo, retrospectivo y de corte transversal. La muestra (no probabilística) fue elegida por conveniencia debido que se tomaron en cuenta la totalidad de los casos que cumplan con los criterios de selección. Se analizaron las tomografías computarizadas de haz cónico seleccionado del archivo del Servicio de Radiología Oral y Maxilofacial de la Unidad de Apoyo al Diagnóstico Estomatológico del Departamento de Odontoestomatología del Hospital Nacional Cayetano Heredia, entre los años 2016 y 2017. En cuanto a los criterios de inclusión, se consideraron las TCHC de pacientes con un rango de edad de 10 a 59 años, de ambos sexos, con presencia de las piezas 34, 35, 36 / 44, 45, 46 y TCHC nítidas, libres de artefactos. Se excluyeron las TCHC con fracturas próximas a la zona de estudio, con patologías tumorales o quísticas en la zona de estudio, de pacientes con malformaciones craneofaciales, y con dispositivos de osteosíntesis en el área de estudio que generen artefactos.

La construcción de variables fue realizada a partir de la definición conceptual del BNM: Configuración morfológica del BNM de acuerdo a los criterios establecidos por Chen et al., (11), esta variable se determinó mediante la evaluación de la TCHC, siendo una variable de tipo cualitativa dicotómica, se consideró como valores: presenta y no presenta. En cuanto a la variable Sexo, su definición conceptual es condición biológica de masculino o femenino. En cuanto a la variable edad, su definición conceptual es el tiempo transcurrido desde el nacimiento hasta la toma de la TCHC y se considera en años y décadas. La variable localización (derecha e izquierda) considera como valores: Unilateral y Bilateral.

Se evaluó la imagen del BNM respecto a su presencia o ausencia y se obtuvo su caracterización. Las observaciones se realizaron en imágenes adquiridas de TCHC según técnica de rutina, con una unidad Rotograph EVO 3D utilizando los parámetros de exposición recomendados por el fabricante: $60-86 \mathrm{kV}$, 6-12 mA; FOV, 8.5x8.5 cm; tamaño del vóxel de 0,25 $\mathrm{mm}$, tamaño del pixel $127 \mu \mathrm{m}$, tiempo de exposición de 20 segundos para una dentición completa.

Los datos de la imagen de corte resultante se convirtieron en imágenes tridimensionales de

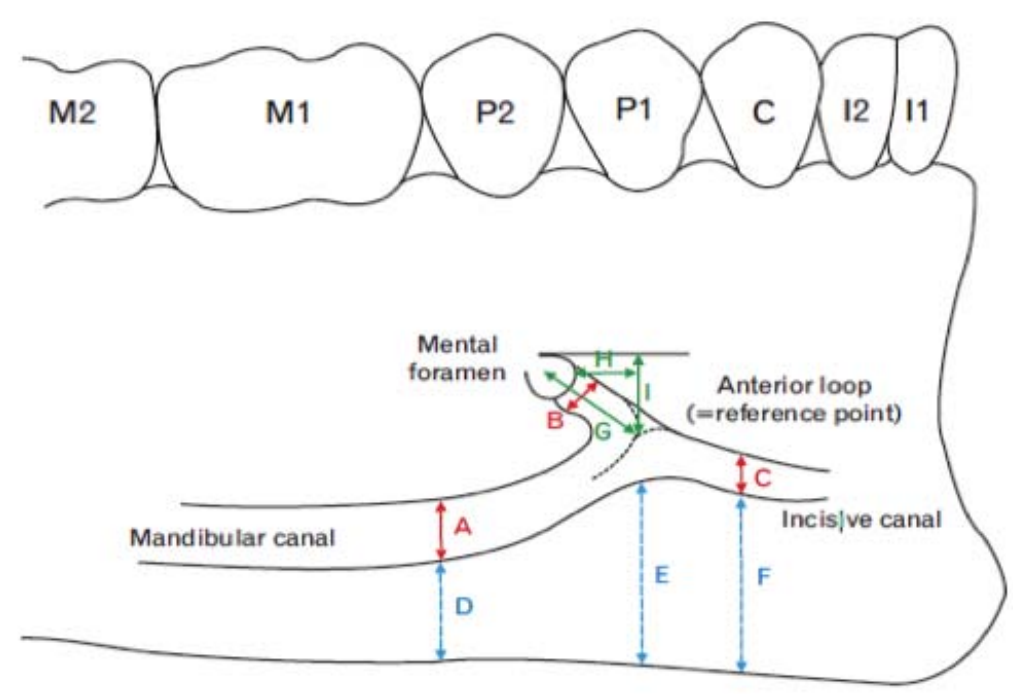

Figura 1. Diagrama mostrando las dimensiones de la medida del bucle anterior (19). 
tomografía computarizada en formato DICOM $®$ y se evaluaron en el Software Dental Estudio Plus. En la reconstrucción panorámica, el nervio fue reconstruido a lo largo del canal del nervio alveolar inferior reconstruido desde la vista axial se utilizó para medir la longitud del BNM (figura 2). La longitud se obtuvo por la distancia entre el borde más anterior del FM hasta el borde más anterior del BNM.

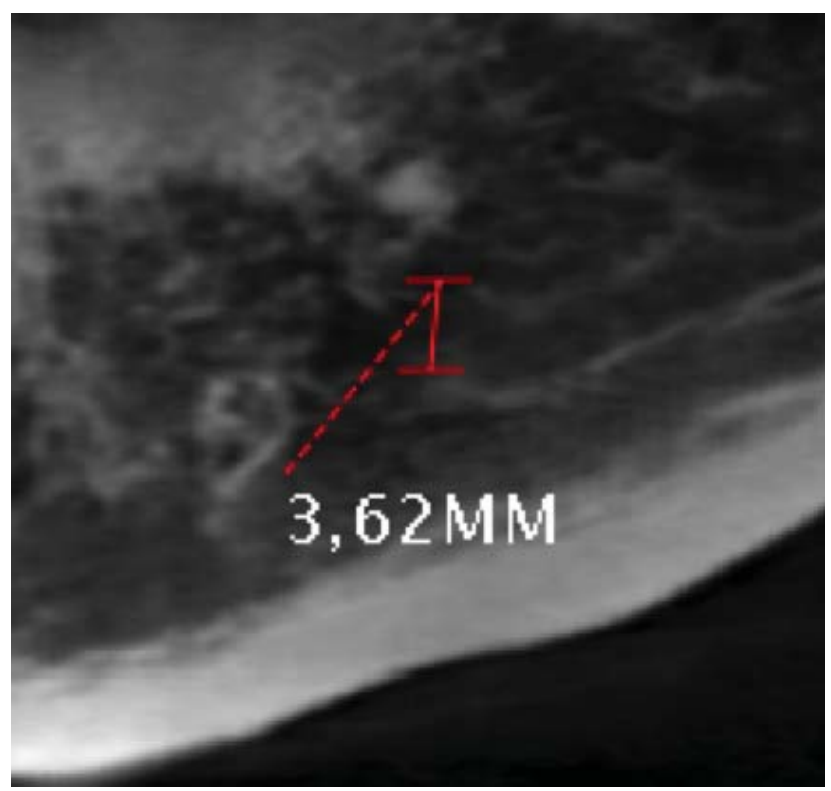

Figura 2. Reconstrucción panorámica mostrando las dimensiones del BNM. (Imagen tomada de la base de datos de TCHC del Hospital Cayetano Heredia)

Las tomas han sido realizadas por especialistas radiólogos orales y maxilofaciales, residentes y técnicos debidamente capacitados del Departamento de Odontoestomatología del Hospital Nacional Cayetano Heredia, durante los años 2016 y 2017.

La calibración se realizó con un especialista en Radiología Bucal y Maxilofacial con más de 10 años de experiencia en el área, con $15 \mathrm{TCHC}$, las cuales se seleccionaron al azar. El propósito de esta prueba fue asegurar la reproducibilidad del estudio.

Se utilizó el paquete estadístico SPSS v. 22 IBM para Windows. Los datos estadísticos fueron llevados con un intervalo de confianza de $95 \%$ y un nivel de significancia de 0,05 . La variable cuantitativa de edad se describió a través del promedio, desviación estándar, mínimo y máximo. Las variables cualitativas de sexo, localización, década de vida, BNM se describieron a través de una tabla de distribución de frecuencias.
Para la comparación de la variable localización según sexo, se utilizó la prueba de Chi Cuadrado $\left(\mathrm{X}^{2}\right)$.

El estudio se realizó luego de recibir la aprobación del Comité Institucional de Ética de la Universidad Peruana Cayetano Heredia (CIEI-UPCH) con fecha 29 de enero del 2018 con código SIDISI N ${ }^{\circ} 101879$ y la aprobación del Comité Institucional de Ética del Hospital Cayetano Heredia (CIEI-HCH) con fecha de 26 de febrero del 2018 con código $\mathrm{N}^{\circ}$ 016-018.

El consentimiento informado de los pacientes no fue necesario por tratarse de tomografías las cuales fueron codificadas manteniendo el anonimato de los pacientes, razón por la cual se solicitó la exoneración de revisión al CIE-UPCH, por ser un estudio retrospectivo con análisis de datos obtenidos a través de una base digital, sin implicaciones éticas.

\section{RESULTADOS}

Se revisaron como universo muestral 181 tomografías computarizadas de haz cónico del Servicio de Radiología Oral y Maxilofacial de la Unidad de Apoyo al Diagnóstico Estomatológico del Departamento de Odontoestomatología del Hospital Cayetano Heredia periodo 2016-2017, en las cuales en el 53,6\% (97 pacientes) se halló la presencia del BNM (tabla 1).

De acuerdo a la longitud del BNM, se tuvo una mínima de $0.12 \mathrm{~mm}$ y una máxima de 7,62 $\mathrm{mm}$. La mayor frecuencia de hallazgos se tuvo en la presentación bilateral en un número de 47 , de la cual en el lado derecho se tuvo una media de 3.04 $\mathrm{mm}$ con una desviación estándar de $1,60 \mathrm{~mm}$ y en el lado izquierdo se tuvo una media de $3.29 \mathrm{~mm}$ con una desviación estándar de $1.74 \mathrm{~mm}$. En cuanto a la presentación unilateral, se tiene que el lado izquierdo se observaron 43 casos, en los cuales la longitud media fue de 3,04 mm, con una desviación estándar

Tabla 1. Distribución del hallazgo del BNM en pacientes evaluados con TCHC en el Departamento de Odontoestomatología del HCH periodo 2016-2017.

\begin{tabular}{lcc}
\hline Hallazgo & n & \% \\
\hline No presenta & 84 & 46,4 \\
Presenta & 97 & 53,6 \\
Total & 181 & 100,0 \\
\hline
\end{tabular}


de 1,46 $\mathrm{mm}$ y en el lado derecho se observaron 7 hallazgos, en los cuales la longitud media fue de 2,24 $\mathrm{mm}$, con una desviación estándar de 0,62 mm., (tabla 2).

Del total de la población hubieron 56 pacientes de sexo masculino, de los cuales 25 presentaron el BNM (25,8\% de los 97 hallazgos); la población femenina fue de 125 pacientes, de las cuales 72 tuvieron BNM $(74,2 \%)$. El valor $p$ fue igual a 0.64 lo que indica que no hay diferencias estadísticas entre ambos grupos (tabla 3).

La distribución de la frecuencia del BNM según número y lateralidad, teniendo en cuenta si son unilaterales o bilaterales fue de $50(27,7 \%)$ para un solo lado y de 47 (26,0\%) para el hallazgo bilateral, con un total de 97 hallazgos de BNM en 181 pacientes $(53,6 \%)$. La frecuencia de BNM predominó en el lado izquierdo con un total de 43 casos $(23,8 \%)$, mientras que el lado derecho tuvo 7 casos (39\%). Para efectos estadísticos se consideran solamente los hallazgos unilaterales (tabla 4).

Según sexo, la edad media de los pacientes de sexo masculino fue de 28.08 años, con una desviación estándar de 14.06 años, la edad mínima fue de 12 años y la máxima de 59. Las mujeres obtuvieron una media de 31.89 años con desviación estándar de 12,99 años, la edad mínima fue de 11 años mientras que la edad máxima de 59 años (tabla 5).

En cuanto al hallazgo de BNM en referencia a la década de vida, la mayor frecuencia se obtuvo en la tercera década de vida comprendida entre 20 a 29 años con 28 hallazgos del BNM (28,9\%), en tanto que la menor frecuencia se observó en la sexta década de vida comprendida entre 50 a 59 años con 13 hallazgos $(13,4 \%)$ (tabla 6$)$.

Tabla 2. Distribución de longitud de BNM según localización, de los pacientes evaluados con TCHC en el Departamento de Odontoestomatología del HCH periodo 2016-2017.

\begin{tabular}{llccccc}
\hline Bilateralidad & n & Mínimo & Máximo & Media & $\begin{array}{c}\text { Desviación } \\
\text { estándar }\end{array}$ \\
\hline \multirow{2}{*}{ Unilateral } & Longitud Derecho & 7 & 1.20 & 3.28 & 2.24 & 0.62 \\
& Longitud Izquierdo & 43 & 0.33 & 6.61 & 3.04 & 1.46 \\
\multirow{2}{*}{ Bilateral } & Longitud Derecho & 47 & 0.12 & 6.56 & 3.04 & 1.60 \\
& Longitud Izquierdo & 47 & 1.00 & 7.62 & 3.29 & 1.74 \\
\hline
\end{tabular}

Tabla 3. Distribución de acuerdo a sexo de los pacientes que presentan BNM evaluados con TCHC en el Departamento de Odontoestomatología del HCH periodo 2016-2017.

\begin{tabular}{lcccccc}
\hline \multirow{2}{*}{ Hallazgo } & \multicolumn{2}{c}{ Masculino } & \multicolumn{2}{c}{ Femenino } & \multicolumn{2}{c}{ Totales } \\
\cline { 2 - 7 } & $\mathbf{n}$ & $\mathbf{\%}$ & $\mathbf{n}$ & $\mathbf{\%}$ & $\mathbf{n}$ & $\mathbf{\%}$ \\
\hline No presenta & 31 & 36.9 & 53 & 63.1 & 84 & 46.4 \\
Presenta & 25 & 25.8 & 72 & 74.2 & 97 & 53.6 \\
Total & 56 & 30.9 & 125 & 69.1 & 181 & 100 \\
\hline
\end{tabular}

Tabla 4. Distribución de frecuencia de BNM según número y lateralidad, de los pacientes evaluados con TCHC en el Departamento de Odontoestomatología del $\mathrm{HCH}$ periodo 2016-2017.

\begin{tabular}{llcc}
\hline & Lado & $\mathbf{n}$ & $\mathbf{\%}$ \\
\hline \multirow{4}{*}{ Hallazgos } & Derecho & 7 & 3.9 \\
& Izquierdo & 43 & 23.8 \\
& Bilateral & 47 & 26.0 \\
& Total & 97 & 53.6 \\
\hline
\end{tabular}


En referencia a la distribución según sexo y lateralidad, se tiene 25 hallazgos de BNM en pacientes de sexo masculino ( $25,7 \%$ de 97 hallazgos), en tanto que en las pacientes de sexo femenino se halló una frecuencia de $72(74,2 \%)$. En cuanto a lateralidad, se aprecia que en el sexo masculino, la mayor frecuencia corresponde al lado izquierdo (14 hallazgos, 56,0\%), en tanto que en el sexo femenino se tiene la mayor frecuencia en el hallazgo bilateral (38 hallazgos, 52,8\%) (tabla 7).

Tabla 5. Distribución de edad de acuerdo a sexo en los pacientes que presentan BNM evaluados con TCHC en el Departamento de Odontoestomatología del HNCH periodo 2016-2017.

\begin{tabular}{lllccccc}
\hline Sexo & & & n & Mínimo & Máximo & Media & $\begin{array}{c}\text { Desviación } \\
\text { estándar }\end{array}$ \\
\hline \multirow{2}{*}{ Masculino } & No presenta & Edad & 31 & 10 & 59 & 34.77 & 16.69 \\
& Presenta & Edad & 25 & 12 & 59 & 28.08 & 14.06 \\
\multirow{2}{*}{ Femenino } & No presenta & Edad & 53 & 10 & 59 & 36.28 & 15.62 \\
& Presenta & Edad & 72 & 11 & 59 & 31.89 & 12.99 \\
\hline
\end{tabular}

Tabla 6. Distribución de década de vida de acuerdo a hallazgo en los pacientes que presentan BNM evaluados con TCHC en el Departamento de Odontoestomatología del HNCH periodo 2016-2017.

\begin{tabular}{cccccccc}
\hline \multirow{2}{*}{$\begin{array}{c}\text { Década de } \\
\text { Vida }\end{array}$} & \multicolumn{4}{c}{ Hallazgo de BNM } & \multicolumn{2}{c}{ Total } \\
\cline { 2 - 5 } & $\mathbf{n}$ & $\mathbf{\%}$ & $\mathbf{n}$ & $\mathbf{\%}$ & $\mathbf{n}$ & $\mathbf{\%}$ \\
\hline De 10 a 19 & 19 & 22,6 & 22 & 22,7 & 41 & 22,7 \\
De 20 a 29 & 14 & 16,7 & 28 & 28,9 & 42 & 23,2 \\
De 30 a 39 & 16 & 19,0 & 19 & 19,6 & 35 & 19,3 \\
De 40 a 49 & 10 & 11,9 & 15 & 15,5 & 25 & 13,8 \\
De 50 a 59 & 25 & 29,8 & 13 & 13,4 & 38 & 21,0 \\
Total & 84 & 100 & 97 & 100 & 181 & 100 \\
\hline
\end{tabular}

Tabla 7. Distribución de frecuencia de BNM según sexo y lateralidad, de los pacientes evaluados con TCHC en el Departamento de Odontoestomatología del HNCH periodo 2016-2017.

\begin{tabular}{llccc}
\hline \multirow{2}{*}{ Lado } & \multicolumn{2}{c}{ Sexo } & \multirow{2}{*}{ Total } \\
\cline { 3 - 4 } & & Masculino & Femenino & \\
\hline \multirow{2}{*}{ Derecho } & Recuento & 2 & 5 & 7 \\
& \% dentro de Sexo & $8,0 \%$ & $6,9 \%$ & $7,2 \%$ \\
\multirow{2}{*}{ Izquierdo } & Recuento & 14 & 29 & 43 \\
& $\%$ dentro de Sexo & $56,0 \%$ & $40,3 \%$ & $44,3 \%$ \\
\multirow{2}{*}{ Bilateral } & Recuento & 9 & 38 & 47 \\
& \% dentro de Sexo & $36,0 \%$ & $52,8 \%$ & $48,5 \%$ \\
\cline { 3 - 4 } Total & Recuento & 25 & 72 & 97 \\
& \% dentro de Sexo & $25,7 \%$ & $74,2 \%$ & $100,0 \%$ \\
\hline
\end{tabular}




\section{DISCUSIÓN}

Entender la anatomía quirúrgica detallada del NAI es esencial para que los especialistas puedan prevenir lesiones potenciales al nervio durante las cirugías, tratamiento endodóntico y en la rehabilitación con implantes de la zona del FM (11).

Las variantes anatómicas del conducto dentario inferior, han sido causantes de un inadecuado bloqueo anestésico, iatrogenia y problemas asociados (2), además de la compresión del haz neurovascular, causando pérdida de la sensibilidad y dolor. En consecuencia, el BNM es una variante anatómica importante en el área interforaminal y la posibilidad del hallazgo anatómico podría complicar un procedimiento quirúrgico en el sector antero inferior. La radiografía panorámica es de mucha importancia en odontología y se ha utilizado durante mucho tiempo como el único examen de diagnóstico, pero debido a las limitaciones que presenta no es posible identificar con exactitud el BNM, la cual debe ser complementada con la TCHC para una mejor visualización de la zona del foramen mental.

En este estudio se evaluaron un total de 181 imágenes tomográficas de los pacientes que acudieron al Servicio de Radiología Oral y Maxilofacial de la Unidad de Apoyo al Diagnóstico Estomatológico del Departamento de Odontoestomatología del Hospital Nacional Cayetano Heredia entre los años 2016-2017.

El total de las imágenes tomográficas fueron evaluadas mediante TCHC, lo que nos permitió compararlo con 12 estudios de similar característica. En este estudio se consideró el uso de esta herramienta imagenológica de alto valor diagnóstico, ya que nos brinda una determinación tridimensional para la evaluación de estructuras anatómicas y hueso disponible, con menor dosis de radiación con respecto a la tomografía espiral multicorte del macizo facial, así mismo permitió medir la longitud de los mismos. Se analizó el bucle del nervio mentoniano respecto a su presencia o ausencia, y su caracterización.

En el presente estudio, la frecuencia de personas con BNM fue de 97 que representan el 53.6\%, estos datos nos indica que poco más de la mitad del total de las TCHC evaluados presentaron BNM, hay coincidencia con estudios de Apostolakis et al. (12) quienes evaluaron 93 pacientes e identificando en el $48 \%$ el $\mathrm{BNM}$ en $\mathrm{TCHC}$, es decir en casi la mitad de los casos estaba presente el BNM, Vujanovic et al. (4) compararon la prevalencia del BNM en radiografía panorámica (RP) y en TCHC, donde obtuvieron el valor de $48.8 \%$ en TCHC similar al estudio realizado por Apostolakis. En otros estudios se obtuvieron valores menores, como en el caso de De Oliveira-Santos et al., observaron el BNM en 22\% y $28 \%$ en RP y TCHC respectivamente (12), De Brito et al., evaluaron 90 pacientes con dos observadores independientes en TCHC el BNM se visualizó en $7.7 \%$, teniendo una baja prevalencia en este estudio (13). En cuanto a otros autores como Filo et al., (15), y Gómez et al., (16), obtuvieron valores superiores al $50 \%$, llegando a los valores entre $69,73 \%$ y $97,3 \%$. Las diferencias entre estos estudios se pueden asociar a los grupos étnicos o a la cantidad de tomografías evaluadas, considerando que el número de pacientes evaluados fue relativamente bajo.

En referencia a la longitud, en el presente estudio se tuvo una media de $3.04 \mathrm{~mm}$ con una mínima de $0,12 \mathrm{~mm}$ y una máxima de $7,62 \mathrm{~mm}$. Al respecto, Uchida et al., midieron con TCHC usando cadáveres dando valores de 0,00 hasta $9,0 \mathrm{~mm}$ con un rango de 1,9+/- 1,7 mm (17), al igual que Chen et al., que demostraron que la longitud del bucle anterior de los taiwaneses $(7,61 \pm 1,81 \mathrm{~mm})$ fue significativamente más largo que de los estadounidenses $(6,22 \pm 1,68$ $\mathrm{mm})$ (11), y al igual que Gómez et al., que hallaron una longitud media de $2,26 \mathrm{~mm}(0,0 \mathrm{~mm}$ y 7,0 $\mathrm{mm}$ como máximo)(16). Sin embargo otros autores, encontraron valores menores, como De OliveiraSantos et al., que observaron el BNM mayor a $2 \mathrm{~mm}$ de extensión anterior (13); Apostolakis et al., quienes identificaron una longitud media de $0,89 \mathrm{~mm}(0-5,7$ $\mathrm{mm}$ ), siendo que en el $95 \%$ de los casos de estudio el BNM era menor a $3 \mathrm{~mm}$ (12); Filo et al., con un valor medio de $1,16 \mathrm{~mm}$, con un rango de 0,3 a $5,6 \mathrm{~mm}$, con el $95,81 \%$ de los lados mostrando valores de 0 a $3 \mathrm{~mm}$ (15); de similar forma, Chen et al., hallaron la media de la longitud del BNM de 1,16 mm (18). En este aspecto, las diferencias también se pueden asociar a los grupos étnicos. Por lo tanto, una distancia invariable anterior al foramen mental no es segura, y la longitud del BNM debe determinarse para cada individuo para evitar lesiones al nervio mental. 
Los hallazgos de imagen del BNM fueron más frecuentes en pacientes de sexo femenino, coincidiendo con el estudio de Filo et al., (15), aunque en el presente estudio la razón de estos resultados puede estar en relación a la mayor afluencia de pacientes del sexo femeninos al Servicio de Radiología Oral y Maxilofacial de la Unidad de Apoyo al Diagnóstico Estomatológico del Departamento de Odontoestomatología del $\mathrm{HNCH}$ durante el periodo de estudio, posiblemente por motivos de estética $\mathrm{y}$ porque que las mujeres podrían tener mayor conciencia de prevención y se preocuparían más por su salud oral. El presente estudio evaluó las TCHC de 125 mujeres y 56 varones, en tanto que Filo evaluó 353 mujeres $(50,86 \%)$ y 341 hombres $(49,14 \%)$; como se observa, en el presente estudio hay diferencias en frecuencia mas no significativas, por lo que se espera que en futuros estudios se puedan considerar cantidades iguales para ambos sexos.

La mayor parte de los hallazgos fueron unilaterales, sin embargo no hubo diferencias significativas, a diferencia de Gómez et al., quienes encontraron en su estudio la mayor parte de hallazgos bilaterales con una diferencia significativa (16). Sin embargo los estudios de Kheir et al., indican que no mostraron diferencias estadísticamente significativas entre las longitudes del BNM y los sexos masculino y femenino (el lado derecho $p=0.33$, el lado izquierdo $p=0.62)(21)$.

En referencia a la década de vida, la mayor frecuencia se dio en el grupo de 20 a 29 años (28 hallazgos, $28,9 \%$ ), en tanto que la menor frecuencia se observa en el grupo de 50 a 59 años (13 hallazgos, 13,4\%). Mientras tanto do Nascimento et al., encontraron la mayor entre la segunda y la quinta década de la vida, y la mayor variación en la longitud se observó en la sexta década, concluyen estos autores que no se observaron diferencias significativas en la longitud ( $p$ $=0,696)$ o la prevalencia $(\mathrm{p}=0,650)$ entre los lados derecho e izquierdo (20). Nosotros encontramos que el BNM era más grande en pacientes jóvenes y se reduce a medida que aumenta la edad del individuo, por lo tanto estos amplios rangos de incidencia pueden estar relacionados con la variabilidad anatómica entre individuos asociada con la edad y la dificultad de visualizar las imágenes del BNM en personas a partir de la de la sexta década de vida.

\section{CONCLUSIONES}

De forma general se concluye que en el 53,6\% de los pacientes evaluados con tomografías computarizadas de haz cónico durante el periodo 2016-2017 en el Servicio de Radiología Oral y Maxilofacial de la Unidad de Apoyo al Diagnóstico Estomatológico del Departamento de Odontoestomatología del Hospital Cayetano Heredia se halló el BNM; en cuanto a la longitud, se tuvo una media de $3.04 \mathrm{~mm}$ con una mínima de 0,12 $\mathrm{mm}$ y una máxima de 7,62 $\mathrm{mm}$. Al observar la distribución por sexo, se verifica que en la población femenina se halló un $74,2 \%$ del total de hallazgos, y un $25,8 \%$ para la población masculina. En referencia a la edad según década de vida, la mayor frecuencia se dio en el grupo de 20 a 29 años con 28 hallazgos del BNM $(28,9 \%)$, y la menor frecuencia en el grupo de 50 a 59 años $(13,4 \%)$. Según lateralidad, se observó 50 hallazgos $(27,7 \%)$ para el hallazgo unilateral, y $47(26,0 \%)$ para bilateral, con un predominio al lado izquierdo con 43 casos $(23,8 \%)$. Estos resultados servirán para implementar la búsqueda imagenológica de rutina del BNM con TCHC como una herramienta científica en la toma de decisiones, previo a los tratamientos quirúrgicos y de rehabilitación en la zona del foramen mental.

Declaración de conflictos de interés: El autor declara no tener conflictos de interés.

\section{Correspondencia:}

Roger Condori Cruz

Correo electrónico: roger.condori@upch.pe

\section{REFERENCIAS BIBLIOGRÁFICAS}

1. Jacobs R, Mraiwa N, van Steenberghe D, Gijbels F, Quirynen M. Appearance, location, course, and morphology of the mandibular incisive canal: an assessment on spiral CT scan. Dentomaxillofac Radiol. 2002; 31(5): 322-7.

2. Beltrán V, Cantín M, Fuentes R, Engelke W. Presencia bilateral del canal incisivo de la mandíbula: Una estructura anatómica con relevancia clínica. Int $\mathrm{J}$ Morphol. 2011; 29(2): 543-9.

3. Yu SK, Kim S, Kang SG, et al. Morphological assessment of the anterior loop of the mandibular canal in Koreans. Anat Cell Biol. 2015; 48(1):75-80.

4. Vujanovic-Eskenazi A, Valero-James JM, SánchezGarcés MA, Gay-Escoda C. A retrospective radiographic evaluation of the anterior loop of the 
mental nerve: comparison between panoramic radiography and cone beam computerized tomography. Med Oral Patol Oral Cir Bucal. 2015; 20(2): 239-245.

5. Wismeijer D, van Waas MA, Vermeeren JI, Kalk W. Patients' perception of sensory disturbances of the mental nerve before and after implant surgery: a prospective study of 110 patients. Br J Oral Maxillofac Surg. 1997; 35(4): 254-9.

6. Mardinger O, Chaushu G, Arensburg B, Taicher S, Kaffe I. Anterior loop of the mental canal: an anatomical-radiologic study. Implant Dent. 2000; 9(2): 120-5.

7. Do Couto-Filho CE, de Moraes PH, Alonso MB, HaiterNeto F, Olate S, de Albergaria-Barbosa JR. Accuracy in the diagnosis of the mental nerve loop: A Comparative study between panoramic radiography and cone beam computed tomography. Int J Morphol. 2015; 33(1): 327-32.

8. Fuentes R, Cantin M, Navarro P, Borie E, Beltrán V, Bucchi C. Caracterización de estructuras anatómicas mediante radiografías panorámicas: El foramen mental. Int J Morphol. 2014; 32 (4): 1423-9.

9. Garay I, Olate S. Consideraciones actuales en el estudio imagenológico de las calcificaciones de tejidos blandos en zona de ángulo mandibular. Int J Odontostomat. 2013; 7(3): 455-64.

10. Garay I, Netto HD, Olate S. Soft tissue calcified in mandibular angle area observed by means of panoramic radiography. Int J Clin Exp Med. 2014; 7 (1): 51-6.

11. Chen JC, Lin LM, Geist JR, Chen JY, Chen $\mathrm{CH}$, Chen YK. A retrospective comparison of the location and diameter of the inferior alveolar canal at the mental foramen and length of the anterior loop between American and Taiwanese cohorts using CBCT. Surg Radiol Anat. 2013; 35(1):11-8.

12. Apostolakis D, Brown JE. The anterior loop of the inferior alveolar nerve: prevalence, measurement of its length and a recommendation for interforaminal implant installation based on cone beam CT imaging. Clin Oral Implants Res. 2012; 23(9): 1022-30.
13. De Oliveira-Santos C, Souza PH, de Azambuja S, et al. Assessment of variations of the mandibular canal through cone beam computed tomography. Clin Oral Investig. 2012; 16 (2): 387-93.

14. De Brito AC, Nejaim Y, de Freitas DQ, de Oliveira Santos C. Panoramic radiographs underestimate extensions of the anterior loop and mandibular incisive canal. Imaging Sci Dent. 2016; 46(3): 159-65.

15. Filo K, Schneider T, Locher MC, Kruse AL, Lübbers HT. The inferior alveolar nerve's loop at the mental foramen and its implications for surgery. J Am Dent Assoc. 2014; 145(3): 260-9.

16. Gómez-Roman G, Lautner NV, Goldammer C, McCoy M. Anterior loop of the mandibular canal-a source of possible complications. Implant Dent. 2015; 24(5): 578-85.

17. Uchida Y, Noguchi N, Goto M, et al. Measurement of anterior loop length for the mandibular canal and diameter of the mandibular incisive canal to avoid nerve damage when installing endosseous implants in the interforaminal region: a second attempt introducing cone beam computed tomography. J Oral Maxillofac Surg. 2009; 67(4): 744-50.

18. Chen Z, Chen D, Tang L, Wang F. Relationship between the position of the mental foramen and the anterior loop of the inferior alveolar nerve as determined by cone beam computed tomography combined with mimics. J Comput Assist Tomogr. 2015; 39(1): 86-93.

19. Yu SK, Kim S, Kang SG, et al. Morphological assessment of the anterior loop of the mandibular canal in Koreans. Anat Cell Biol. 2015; 48(1):75-80.

20. do Nascimento EH, Dos Anjos ML, Dos Anjos A, et al. Assessment of the anterior loop of the mandibular canal: A study using cone-beam computed tomography. Imaging Sci Dent. 2016; 46(2): 69-75.

21. Kheir MK, Sheikhi M. Assessment of the anterior loop of mental nerve in an Iranian population using cone beam computed tomography scan. Dent Res J (Isfahan). 2017; 14(6): 418-22.

Recibido: 09-01-2019

Aceptado: 13-05-2019 